\title{
Integrating P2P Energy Trading with Probabilistic Distribution Locational Marginal Pricing
}

\author{
Thomas Morstyn, Member, IEEE, Alexander Teytelboym, Cameron Hepburn and \\ Malcolm D. McCulloch, Senior Member, IEEE
}

\begin{abstract}
This paper proposes a new local energy market design for distribution systems, which integrates peer-to-peer (P2P) energy trading and probabilistic locational marginal pricing. Distribution locational marginal pricing and P2P energy trading have each been proposed as potential alternatives to traditional retail pricing, to improve coordination between prosumers with distributed energy resources. Unidirectional locational pricing provides a scalable approach for coordinating demand, considering constraints and losses; while P2P energy trading allows prosumers to negotiate mutually beneficial bilateral energy transactions that increase the utilisation of their flexible energy resources. This paper proposes a market design combining the benefits of these two strategies. First, a new strategy for dayahead locational marginal pricing is developed, which manages the uncertainty associated with local generation, demand and upstream prices by introducing a spread between the prices charged for energy imports and paid for energy exports. Then, local P2P energy trading platforms are integrated to additionally enable direct prosumer-to-prosumer trading, with transaction fees penalising energy transfers according to probabilistic differential locational marginal prices. Case studies are presented for a multi-phase low voltage distribution network, showing how the design can create value for prosumers, and the system as a whole, by reducing the curtailment of renewable generation.
\end{abstract}

Index Terms-Distribution locational marginal pricing, distribution system operator, local energy market, peer-to-peer energy trading, point estimate method, transactive energy.

\section{INTRODUCTION}

$\mathbf{T}$ HIS paper proposes a new local energy market design for distribution systems, which integrates peer-to-peer (P2P) energy trading and probabilistic distribution locational marginal prices (DLMPs). The proposed market design enables prosumers to directly negotiate bilateral energy transactions that account for network constraints and uncertainty, without requiring a central operator to check and approve transactions.

Energy markets are undergoing a fundamental transition due to the emergence of 'prosumers' within electricity distribution networks [1]. Prosumers are small-scale consumers with distributed energy resources (DERs), such as renewable sources

This work is supported by the Engineering and Physical Sciences Research Council (award references EP/S000887/1 and EP/S031901/1).

T. Morstyn and M. D. McCulloch are with the Department of Engineering Science at the University of Oxford, Oxford OX1 2JD, United Kingdom (email: thomas.morstyn@eng.ox.ac.uk, malcolm.mcculloch@eng.ox.ac.uk)

A. Teytelboym is with the Department of Economics at the University of Oxford, Oxford OX1 2JD, United Kingdom (email: alexander.teytelboym@economics.ox.ac.uk).

C. Hepburn is with the Smith School for Enterprise and Environment at the University of Oxford, Oxford OX1 2JD, United Kingdom (email: cameron.hepburn@smithschool.ox.ac.uk). and home batteries, along with smart meters and energy management systems that enable them to proactively manage their energy demand. If properly coordinated, prosumer-owned DERs could offer significant value by reducing losses and alleviating network constraints which might otherwise require renewable curtailment or costly infrastructure upgrades [2].

Existing energy market arrangements do not facilitate active coordination within distribution networks. Prosumers are too small to be integrated into wholesale market dispatch, and instead are serviced in the retail market with long term contracts that specify a fixed energy price or a set of timeof-use prices [3]. Retail time-of-use pricing helps coordinate DERs relative to aggregate demand at the transmission system level, but does not account for local demand conditions [4].

This has led to interest in local energy markets to incentivise coordination between distribution network prosumers. In this paper, the entity responsible for arranging a local energy market is referred to as the distribution system operator (DSO). Local energy market designs can be broadly divided into designs based on (i) centralised dispatch [5]-[8]; (ii) distributed dispatch [9]-[14]; (iii) unidirectional pricing [15][17]; and (iv) P2P energy trading [18]-[27].

Local energy market designs based on centralised dispatch require prosumers to provide sufficient information to the DSO for their DERs to be directly scheduled (e.g. generation/demand bid curves and constraints) [5]-[8]. The DSO can solve an optimal power flow (OPF) problem to calculate an efficient and feasible schedule for the DERs. The dual variables associated with the OPF energy balance constraints at each bus can be interpreted as DLMPs [28]. The DLMPs are a desirable pricing mechanism, since they satisfy allocative efficiency, meaning that they price demand at the marginal cost of supply [29]. Alternative pricing strategies based on cooperative game theory and fairness criteria have also been proposed [30]-[32].

Distributed dispatch strategies remove the need for the DSO to collect detailed prosumer information, instead making use of iterative negotiation and local decision making [9]-[14]. A range of iterative market mechanisms have been proposed including Stackelberg games, where the DSO pursues profitmaximisation [9], [10]; mean-field games, where the DSO seeks to flatten aggregate demand [11], [12]; and distributed optimisation [13], [14]. Distributed optimisation based on dual price variable adjustment (e.g. dual decomposition, alternating direction method of multipliers) resembles an auction process, and can be used to find the centralised dispatch solution and associated DLMPs. However, to achieve convergence these 
mechanisms generally require prosumers to cooperatively augment their objective functions with penalty terms, rather than making purely utility-maximising decisions [33].

Scalability is a concern for distribution system dispatch, since the complexity increases with the number of DERs. Distributed designs can help improve scalability compared with centralised designs by taking advantage of parallel processing. However, the number of iterations required to reach convergence increases with the number of resources, which means that at larger scales the communications overhead will dominate the computation time [34]. Coordinating the vast number of DERs that are expected within future power systems will require at least partial decoupling between coordination mechanisms at different physical scales [35].

An alternative approach, which decouples distribution system dispatch from small-scale resource coordination, is to calculate DLMPs based on day-ahead forecasts and send these as unidirectional price signals to prosumers [15]-[17]. This approach is based on the observation that the DLMPs are compatible with incentivising individual prosumer decisions that match the centralised dispatch solution [29]. However, directly implementing DLMPs calculated from the day-ahead OPF problem has two limitations. First, in the case of inaccurate forecasts the DLMPs will not incentivise efficient operation. Second, even with accurate forecasts, if the optimal dispatch has multiple solutions (known as dual degeneracy), unidirectional pricing is not guaranteed to incentivise decisions which are collectively efficient or even feasible [36]. For example, consider a system with multiple storage systems which are scheduled to export power across several time intervals. If DLMPs are sent to the storage system owners unidirectionally, they may individually select the same interval to export energy, resulting in network constraint violations.

Recently, there has been interest in local energy market designs based on P2P energy trading, which would allow prosumers to directly negotiate energy transactions with one another [18]-[27]. P2P energy trading offers advantages in terms of prosumer autonomy, the ability to express individual preferences, market transparency and competition [37].

Dealing with network constraints is challenging without reintroducing a centralised mechanism. In [21], P2P transaction fees based on the electrical distance between distribution network zones are introduced. The design in [38] introduces a separate market for distribution system flexibility, where a DSO can incentivise prosumers within a certain zone to reduce their scheduled demand. These designs only indirectly address network constraints, which depend on the network topology between the prosumers. In [22] and [23], iterative mechanisms are introduced that place the DSO in the loop to check the feasibility of P2P market outcomes. In the case of infeasibility, the DSO either blocks certain transactions or updates transaction fees, and directs the prosumers to engage in a new round of $\mathrm{P} 2 \mathrm{P}$ negotiation. These approaches address the need to manage distribution network constraints, but reintroduce the DSO as a central authority, limiting scalability and market transparency.

The local energy market design proposed in this paper integrates P2P energy trading platforms into a distribution network with unidirectional locational pricing. The proposed market design incorporates network constraints, losses, demand uncertainty and upstream price uncertainty, without requiring a DSO to check and approve transactions. First, a new probabilistic strategy for day-ahead locational pricing is developed, which manages uncertainty by introducing a spread between locational import and export prices. Then, local P2P energy trading platforms are integrated to additionally enable multi-period day-ahead P2P trading and single-period intraday P2P trading, with transaction fees penalising energy transfers according to the probabilistic differential DLMPs. Case study simulations are presented showing how the proposed strategy can address voltage constraints within a multi-phase low voltage distribution network, and how introducing P2P trading can create value for prosumers, and the system as a whole, by reducing the curtailment of renewable generation.

The novel contribution of the paper has three main components:

1) A novel DSO pricing strategy is proposed based on dayahead three-phase probabilistic dispatch. The method is scalable since prices are calculated day-ahead and sent unidirectionally to prosumers. It is shown that by increasing the spread between import and export prices, (i) the potential for network constraint violations is reduced, despite load/generation uncertainty and dual degeneracy; and (ii) the likelihood that the DSO's revenue will not cover its costs is reduced, despite upstream price uncertainty.

2) A novel strategy is proposed for managing three-phase network constraints within P2P energy trading markets that involve purely bilateral negotiation and fully autonomous decision making by prosumers seeking to maximise their utility. This extends the authors' previous work from [18], which proposed a purely bilateral P2P market design, but did not address network constraints. Unlike existing approaches, a central authority is not introduced in the loop to check and approve transactions. Instead, probabilistic transaction fees posted day-ahead incentivise feasible outcomes, without compromising scalability and prosumer autonomy.

3) A novel local energy market design is proposed which combines these two strategies. It is shown that by enabling local P2P energy trading between prosumers subject to the DSO pricing strategy, not only do the prosumers achieve higher utility, but that overall economic efficiency can also be improved.

The rest of this paper is organised as follows. Section II presents the structure of the proposed market design. In Section III, the new strategy for unidirectional pricing based on probabilistic DLMPs is developed, and in Section IV, it is shown how P2P energy trading can be incorporated. Simulation case studies are presented in Section V. Section VI concludes the paper.

\section{Market Structure}

This paper considers a distribution network with a DSO that is responsible for setting day-ahead locational import and export prices and P2P transaction fees. Local P2P energy 


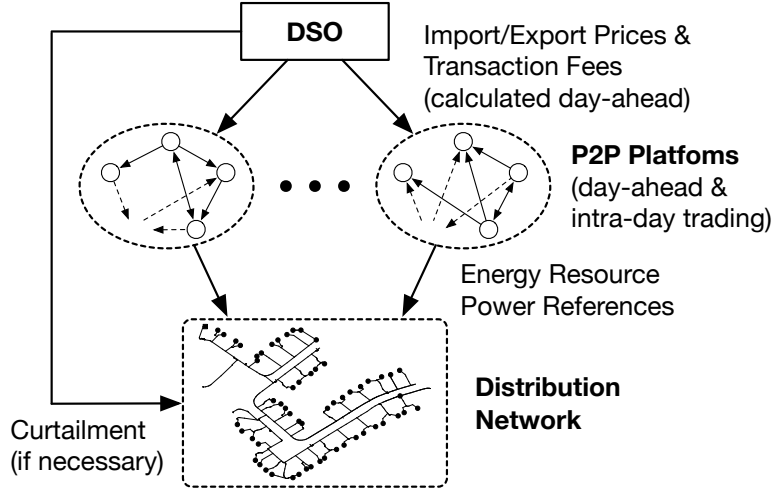

Fig. 1: High level block diagram of the proposed local energy market design.

trading platforms allow prosumers to negotiate bilateral energy transactions. Fig. 1 shows a high level block diagram of the proposed market design.

Under the proposed market design, the DSO estimates probabilistic DLMPs associated with day-ahead dispatch, considering the uncertainty associated with upstream energy prices, local renewable generation and local demand. The DSO then sets prices that prosumers are charged for energy imports, and prices that they are paid for energy exports, based on the mean values of the DLMPs and a spread calculated based the DLMP standard deviations and a 'price-spread scale factor'. A larger price spread increases the likelihood the DSO will operate with positive revenue, despite the uncertainty of upstream energy prices. The price spread also helps reduce the likelihood that network constraints will be violated, since prosumers are incentivised to use local flexibility to reduce their net demand/generation, e.g. by shifting local generation to reduce demand, or by curtailing excess generation. These prices are sent to prosumers unidirectionally, as a scalable mechanism for coordination. Under the proposed market design, the DSO will honour the prices which it sends to the prosumers. This means that the DSO is exposed to upstream price uncertainty, but the prosumers are not. Increasing the price-spread between imports and exports increases the likelihood that the DSO's revenue will cover its costs, which is necessary for sustainable operation.

The price spread incentivises prosumers to increase their individual self-consumption, and therefore would incentivise conservative DER utilisation without additional market mechanisms. This is addressed by allowing local P2P energy trading platforms to operate within the distribution network. This enables the prosumers to additionally engage in multiperiod day-ahead P2P trading based on their expected generation/demand, and during operation to engage in single-period P2P trading based on their updated (e.g. 30 minute ahead) generation/demand predictions and day-ahead market obligations. Conservative transaction fees are set by the DSO based on probabilistic differential DLMPs to reduce the likelihood that P2P trading will result in network constraint violations. During operation, the DSO curtails DERs it can directly manage (e.g. community-scale PV plants) if necessary to ensure network constraints are not violated.

The imposition of the P2P transaction fees posted day-ahead by the DSO will influence how the prosumers can exercise their flexibility. However, the local P2P trading platforms still achieve desirable objectives including: (i) scalability, since $\mathrm{P} 2 \mathrm{P}$ negotiation is based purely on communication between trading partners, with prosumers making individual decisions in parallel; and (ii) prosumer autonomy, since at each step of negotiation, prosumers make autonomous decisions to maximise their individual utility.

The spread between import and export prices is controlled by the price-spread scale factor. Since the DSO operates as a regulated monopoly, the electricity market regulator would need to specify the scale factor, or limit its allowable range, so that the DSO is able to make sufficient profits for sustainable operation, without making excessive profits at the expense of prosumers. The paper does not propose a particular value for the scale factor. Instead, Section V shows how varying the price-spread scale factor impacts network constraints; the relative profits of the DSO and the prosumers; and overall utility, with and without $\mathrm{P} 2 \mathrm{P}$ energy trading. The introduction of a spread between import and export prices is inspired by retail energy pricing, where excess supply is commonly remunerated at a lower 'feed-in price', incentivising behindthe-meter self-consumption [39].

\section{Probabilistic Distribution Locational MARGINAL PRICING}

In this section, a multi-phase multi-period day-ahead distribution system OPF problem is formulated. Then, the mean and standard deviation values of the DLMPs and differential DLMP probability distributions are estimated and a conservative strategy for unidirectional pricing is proposed.

\section{A. Day-Ahead Multi-Phase Distribution System Dispatch}

The DSO uses a multi-period day-ahead distribution system OPF problem as the basis for calculating probabilistic DLMPs. It is assumed that the DSO is operating in a regulatory environment which gives it the objective of maximising overall social welfare. To achieve this, the dispatch problem should account for upstream energy prices, network constraints, and the prosumers' costs and constraints.

Let $\mathcal{T}=\{1, \ldots, T\}$ be the set of optimisation intervals, each of duration $\Delta t$. The distribution network has buses $\mathcal{N}=$ $\{0, \ldots, N\}$, where bus 0 is the slack bus. The network has three phases, $\Phi=\{a, b, c\}$. Let $Y \in \mathbb{C}^{3(N+1) \times 3(N+1)}$ be the three-phase admittance matrix. The admittance matrix can be partitioned into $Y=\left[\begin{array}{cc}Y_{00} & Y_{0 N} \\ Y_{N 0} & Y_{N N}\end{array}\right]$, where $Y_{00} \in \mathbb{C}^{3 \times 3}$.

Let $\mathcal{P}=\{1, \ldots, P\}$ be the set of prosumers in the distribution network. It is assumed that each prosumer has some combination of an inflexible load, a PV source and an energy storage system. The storage systems are modelled with a linear relationship between output power and energy stored, and degradation costs that are proportional to energy throughput, which is a standard approach for convex optimisation [40]. The fixed-point linearisation from [41] is used to obtain a linear formulation for the network constraints. 
The multi-period day-ahead distribution system OPF problem can formulated as a linear program

$$
\begin{aligned}
& \min \sum_{t \in \mathcal{T}} \Delta t\left(\hat{\lambda}_{0 t} \sum_{\phi \in \Phi} p_{0 t}^{\phi}+\sum_{i \in \mathcal{P}} c_{i}^{d e g}\left(p_{i t}^{c h}+p_{i t}^{d i s}\right)\right) \\
& \text { s.t. } 0 \leq p_{i t}^{p v} \leq \hat{\bar{p}}_{i t}^{p v}, 0 \leq p_{i t}^{c h} \leq \bar{p}_{i t}^{c h}, 0 \leq p_{i t}^{d i s} \leq \bar{p}_{i t}^{d i s} \\
& \underline{E}_{i t} \leq \sum_{t=1}^{\tau} \Delta t\left(\eta_{i}^{c h} p_{i t}^{c h}-\frac{p_{i t}^{d i s}}{\eta_{i}^{d i s}}\right)+E_{i 0} \leq \bar{E}_{i t}, \tau \in \mathcal{T}, \\
& \sum_{t \in \mathcal{T}} \Delta t\left(\eta_{i}^{c h} p_{i t}^{c h}-\frac{p_{i t}^{d i s}}{\eta_{i}^{d i s}}\right)=0 \\
& p_{i t}^{n e t}=\hat{p}_{i t}^{l o a d}-p_{i t}^{p v}+p_{i t}^{c h}-p_{i t}^{d i s} \\
& p_{l t}=-\sum_{i \in \mathcal{P}} \mathcal{M}_{l i} p_{i t}^{n e t} \\
&|\underline{v}| \leq K_{t}\left(p_{t}-\tilde{p}_{t}\right)+\left|\tilde{v}_{t}\right| \leq|\bar{v}|, \\
& p_{0 t}=G_{t}\left(p_{t}-\tilde{p}_{t}\right)+\tilde{p}_{0 t}, \\
& K_{t}=\operatorname{diag}\left(\left|\tilde{v}_{t}\right|\right)^{-1} \operatorname{Real}\left\{\operatorname{diag}\left(\tilde{v}_{t}^{*}\right) Y_{N N}^{-1} \operatorname{diag}\left(\tilde{v}_{t}^{*}\right)^{-1}\right\} \\
& G_{t}=\operatorname{Real}\left\{\operatorname{diag}\left(v_{0 t}\right) Y_{0 L}^{*}\left(Y_{N N}^{-1} \operatorname{diag}\left(\tilde{v}_{t}^{*}\right)^{-1}\right)^{*}\right\} .
\end{aligned}
$$

Here, for simplicity, it is assumed prosumers have wye connections, that PV sources and energy storage systems operate with unity power factor and only network voltage constraints are included. Reactive power decision variables, delta connected devices and line constraints can be incorporated using equations from [41]. The decision variables are the energy storage system charging and discharging powers $p_{i t}^{c h}, p_{i t}^{d i s}$ and the PV generation $p_{i t}^{p v}$ for each prosumer $i \in \mathcal{P}$ and interval $t \in \mathcal{T}$.

The objective (1a) is to minimise the net cost of energy traded upstream and battery degradation. $\hat{\lambda}_{0 t}$ is the predicted price of energy at the grid connection point and $p_{0 t}^{\phi}$ is the real power import on phase $\phi$ at the grid connection point $\left(p_{0 t}^{\phi}<0\right.$ indicates real power is exported). $c_{i}^{d e g}>0$ is the cost of degradation associated with energy throughput for prosumer $i$ 's energy storage system. The objective (1a) implicitly assumes that the local market is small relative to the upstream wholesale market, and therefore that upstream energy prices are not directly impacted by local demand. A simplified approach for modelling the impact of local demand on upstream energy prices would be to include an additional term $f_{0 t}\left(\sum_{\phi \in \Phi} p_{0 t}^{\phi}\right)$, where $f_{0 t}(\cdot)$ is a convex function modelling the increased cost of obtaining energy upstream as the net demand increases due to wholesale market merit order dispatch (see e.g. [42]).

The prosumer operating constraints are given by (1b)-(1e). For prosumer $i$ and interval $t$, the predicted available PV generation is given by $\hat{\bar{p}}_{i t}^{p v}$. The energy storage system output power is limited to the range $\left[-\bar{p}_{i t}^{c h}, \bar{p}_{i t}^{d i s}\right]$, and the energy stored is limited to $\left[\underline{E}_{i t}, \bar{E}_{i t}\right]$. Constraint (1d) requires the final energy level to match the initial energy level $E_{i 0}$. The energy storage system charging efficiency is $\eta_{i}^{c h} \in(0,1]$ and the discharging efficiency is $\eta_{i}^{\text {dis }} \in(0,1]$. Constraint (1e) defines prosumer $i$ 's net demand $p_{i t}^{\text {net }}$, where $\hat{p}_{i t}^{\text {load }}$ is the predicted inflexible load. Note that for each $t \in \mathcal{T}$ and $i \in \mathcal{P}$, at least one of $p_{i t}^{c h}$ and $p_{i t}^{d i s}$ will equal zero, since, if this wasn't the case, there would be a feasible lower cost solution with one at zero and the other at a lower value [8].

The network constraints are given by (1f)-(1h). To obtain a linear power flow model, the nonlinear power flow is solved using the Z-bus method [43], for intervals across the day for nominal operation with $p_{i t}^{c h}=0, p_{i t}^{d i s}=0, p_{i t}^{p v}=\hat{\bar{p}}_{i t}^{p v}$. For nominal operation, $\left|\tilde{v}_{t}\right|=\left(\left|\tilde{v}_{1 t}\right|, \ldots,\left|\tilde{v}_{N t}\right|\right)$ is the vector of bus voltage magnitudes, where $\tilde{v}_{l t}=\left(\tilde{v}_{l t}^{a}, \tilde{v}_{l t}^{b}, \tilde{v}_{l t}^{c}\right)$. $\tilde{p}_{0 t}=\left(\tilde{p}_{0 t}^{a}, \tilde{p}_{0 t}^{b}, \tilde{p}_{0 t}^{c}\right)$ is the vector of real power imports at the grid connection point. The vector of net power injected at each bus and phase is given by $p_{t}$, where $p_{t}=\left(p_{1 t}, \ldots, p_{N t}\right)$, and $p_{l t}=\left(p_{l t}^{a}, p_{l t}^{b}, p_{l t}^{c}\right)$, and $\tilde{p}_{t}$ are the nominal injections. $\mathcal{M}_{l i} \in \mathbb{R}^{3}$ relates prosumer $i$ 's predicted net demand to the demand on the phases of bus $l$ (e.g. $\mathcal{M}_{l i}=\left(\frac{1}{3}, \frac{1}{3}, \frac{1}{3}\right)$ for a balanced 3-phase connection and $(1,0,0)$ for a single $a$-phase connection). $|\bar{v}|,|\underline{v}|$ are vectors of upper and lower voltage limits.

The DLMPs can be obtained from the dual variables associated with the solution of (1) [29]. Let $\nu_{t}^{p *}, \nu_{t}^{|\bar{v}| *}, \nu_{t}^{|\underline{v}| *}, \nu_{t}^{p_{0} *}$ be the vectors of dual variables associated with constraints (1f)(1h), at the optimal solution. Then, the vector of DLMPs is given by

$$
\lambda_{t}=\frac{1}{\Delta t}\left(\nu_{t}^{p *}+G_{t}^{\top} \nu_{t}^{p_{0} *}+K_{t}^{\top}\left(\nu_{t}^{|\bar{v}| *}-\nu_{t}^{|\underline{v}| *}\right)\right)
$$

where $\lambda_{t}=\left(\lambda_{1 t}, \ldots, \lambda_{N t}\right)$ and $\lambda_{l t}=\left(\lambda_{l t}^{a}, \lambda_{l t}^{b}, \lambda_{l t}^{c}\right) . \lambda_{l t}^{\phi}$ specifies the marginal cost of supplying an increase in the energy demand on phase $\phi$ of bus $l$ during interval $t$, accounting for the losses and active network constraints associated with the optimal solution. The differential DLMP $\Delta \lambda_{l m t}^{\phi \psi}=\lambda_{m t}^{\psi}-\lambda_{l t}^{\phi}$ gives the marginal cost of an energy transfer from phase $\phi$ of bus $l$ to phase $\psi$ of bus $m$ during interval $t$.

\section{B. Point Estimate Method}

The DLMPs are uncertain, since the day-ahead OPF problem depends on variable upstream energy prices, the available PV generation and the prosumers' inflexible loads. Note that even using state-of-the-art forecasting methods, there is a significant level of uncertainty associated with load forecasts at the low voltage distribution network level [44]. Here, the point estimate method from [45] is used to estimate the means and variances of the DLMPs. Point estimate methods were previously proposed for estimating probabilistic LMP distributions in [46]. The advantages of point estimate methods are limited computational burden, the ability to account for correlations between sources of uncertainty and the applicability to general model functions.

It is assumed the DSO is able to model the sources of uncertainty with random variables (e.g. using historical data). Random variables are introduced for each time interval to model the upstream energy prices $\hat{\lambda}_{0 t}, t \in \mathcal{T}$. It is assumed the physical proximity of PV sources in the distribution network means their available generation is uniform at each interval. Therefore, the PV generation uncertainty can be modelled by a single random variable for each time interval $\hat{x}_{t}^{p v}, t \in \mathcal{T}$, which is multiplied by the capacity of each prosumer's PV source $\bar{p}_{i}^{p v}, i \in \mathcal{P}$ to obtain the individual available $\mathrm{PV}$ generation $\hat{\bar{p}}_{i t}^{p v}=\hat{x}_{t}^{p v} \bar{p}_{i}^{p v}, i \in \mathcal{P}, t \in \mathcal{T}$. In general, the full prosumer load variability could be modelled with random variables introduced for each prosumer and interval. To reduce the number of random variables, each prosumer is assigned a normalised load profile based on historical data $n_{i t}^{\text {load }}, t \in \mathcal{T}$, 
and their total energy demand over the day is treated as a random variable $x_{i}^{\text {load }}, i \in \mathcal{P}$ which scales this normalised load profile, so that $\hat{p}_{i t}^{\text {load }}=\hat{x}_{i}^{\text {load }} n_{\text {it }}^{\text {load }} / \Delta t, t \in \mathcal{T}$. This approach only requires one random variable per prosumer, reducing the computational burden, with the trade-off of not accounting for the full range of potential load variability.

The Appendix describes how the point estimate method is used to estimate the means and standard deviations of the DLMPs $\mu_{\lambda_{l t}^{\phi}}, \sigma_{\lambda_{l t}^{\phi}}$ and differential DLMPs $\mu_{\Delta \lambda_{l m t}^{\phi \psi}}, \sigma_{\Delta \lambda_{l m t}^{\phi \psi}}$. Uncertainty associated with the upstream energy prices, PV generation and prosumer inflexible loads have been considered, since these vary during operation. In this paper, it has been assumed that DER parameters which do not change over short timescales are known by the DSO. This information could be obtained using parameter estimation methods (see e.g. [47]), or in future from a DER asset register [48], [49]. Alternatively, since the point estimate method can be used for general model functions, it can be straightforwardly extended to account for additional sources of uncertainty. For example, if the DSO did not know the degradation cost of prosumer $i$ 's battery, but knew an interval $\left[\underline{c}_{i}^{d e g}, \bar{c}_{i}^{d e g}\right]$ over which it was likely to fall, it could be modelled as a uniform random variable, $c_{i}^{\text {deg }} \sim \mathcal{U}\left(\underline{c}_{i}^{\text {deg }}, \bar{c}_{i}^{\text {deg }}\right)$, rather than as a deterministic quantity.

\section{DSO Pricing Strategy}

Under the proposed market design, the DSO sets day-ahead locational prices, with a spread between the price charged for importing energy and the price paid for exporting energy. These are sent to the prosumers in a unidirectional manner. The import and export prices for phase $\phi$ of bus $l$ during interval $t$ are given by

$$
\begin{aligned}
& \lambda_{l t}^{i m p, \phi}=\mu_{\lambda_{l t}^{\phi}}+\alpha \sigma_{\lambda_{l t}^{\phi}}, \\
& \lambda_{l t}^{e x p, \phi}=\mu_{\lambda_{l t}^{\phi}}-\alpha \sigma_{\lambda_{l t}^{\phi}} .
\end{aligned}
$$

The price-spread scale factor $\alpha \geq 0$ is selected by the DSO and sets the magnitude of the price spread relative to the standard deviations of the DLMPs. For each time interval, the price of importing energy on a particular phase and bus is given by the mean value of the associated DLMP, plus its standard deviation multiplied by the price-spread scale factor (DLMP means and standard deviations are obtained from the point estimate method). The export price is given by the mean, minus the standard deviation multiplied by the scale factor. This means that when the price-spread scale factor is zero. the prices of imports and exports are both equal to the mean DLMP values. When the price-spread scale factor is high, prices are conservative, with import prices likely to be higher than the DLMPs that would result from centralised dispatch, and the export prices likely to be lower.

The import and export prices prosumers are exposed to depend on their network connections. Assuming prosumer $i$ is connected at bus $l$, the equivalent import and export prices during interval $t$ are given by

$$
\begin{aligned}
\lambda_{i t}^{i m p} & =\mathcal{M}_{l i}^{\top}\left(\lambda_{l t}^{i m p, a}, \lambda_{l t}^{i m p, b}, \lambda_{l t}^{i m p, c}\right), \\
\lambda_{i t}^{e x p} & =\mathcal{M}_{l i}^{\top}\left(\lambda_{l t}^{\text {exp }, a}, \lambda_{l t}^{\text {exp }, b}, \lambda_{l t}^{e x p, c}\right) .
\end{aligned}
$$

Network constraints and losses are accounted for using P2P transaction fees set ahead of operation by the DSO. If the DSO had fully accurate forecasts, DLMPs from a deterministic dispatch problem would be compatible with incentivising efficient operation among prosumers pursuing utility-maximisation, due to the incentive compatibility property of locational marginal pricing [29]. The difference between the DLMPs of two nodes would be equal to the marginal network cost associated an additional P2P transaction between them. Under the proposed market design, uncertainty is addressed by using conservative P2P transaction fees which depend on both the mean and standard deviation values of the differential DLMPs between nodes. The means and standard deviations are obtained using the point estimate method. The transaction fee associated with energy transfers from phase $\phi$ of bus $l$ to phase $\psi$ of bus $m$ during interval $t$ is given by

$$
\delta_{l m t}^{\phi \psi}=\max \left\{\mu_{\Delta \lambda_{l m t}^{\phi \psi}}+\alpha \sigma_{\Delta \lambda_{l m t}^{\phi \psi}}, 0\right\} .
$$

Here, a conservative restriction is imposed that $\mathrm{P} 2 \mathrm{P}$ transaction fees are greater than or equal to zero. Although day-ahead differential DLMPs may be negative, in practice offering a subsidy on $\mathrm{P} 2 \mathrm{P}$ transactions can result in prosumers over provisioning these transactions, which can be costly for the DSO. For an energy transfer from prosumer $i$ at bus $l$ to prosumer $j$ at bus $m$ during interval $t$, the equivalent $\mathrm{P} 2 \mathrm{P}$ transaction fee they are exposed to is given by

$$
\delta_{i j t}=\mathcal{M}_{l i}^{\top}\left[\begin{array}{lll}
\delta_{l m t}^{a a} & \delta_{l m t}^{a b} & \delta_{l m t}^{a c} \\
\delta_{l m t}^{b a} & \delta_{l m t}^{b b} & \delta_{l m t}^{b c} \\
\delta_{l m t}^{c a} & \delta_{l m t}^{c b} & \delta_{l m t}^{c c}
\end{array}\right] \mathcal{M}_{m j}
$$

Under the proposed market design, the DSO sets import and export prices on a day-ahead basis. This removes the need for the DSO to directly dispatch resources or approve transactions during operation. The price-spread scale factor needs to be selected using historical data in anticipation of real time operation to balance different market objectives. As will be shown in Section V, a higher price-spread scale factor will increase DSO revenue, and will incentivise prosumers to operate their DERs to reduce the maximum power which they import and export. Therefore, a scale factor should be selected which is high enough so that the DSO will have positive net revenue, which is necessary for sustainable operation, and the likelihood of network constraint violations is reduced, but which is not so high that overall efficiency is significantly reduced due to prosumer DERs being operated in an overly conservative manner.

\section{Peer-to-Peer Energy Trading}

In this section, it is shown how $\mathrm{P} 2 \mathrm{P}$ energy trading platforms can be integrated into the local energy market design. Prosumers engage in a multi-period day-ahead market where they trade discrete bilateral forward contracts and schedule their energy storage systems based on renewable generation/demand predictions. During operation, single-period P2P markets allow prosumers to trade based on updated predictions and dayahead P2P obligations. Under the proposed market design, prosumers must fulfil contracts negotiated in the day-ahead market during operation. This can be done using their own 
energy resources, by trading with peers, or by trading with the DSO. For example, if a prosumer had sold a day-ahead P2P contract, but during operation the prosumer did not have sufficient generation to satisfy it, they would need to buy energy to cover their energy delivery obligation. Multiple $\mathrm{P} 2 \mathrm{P}$ platforms can operate in parallel within the distribution network, coordinated by the locational prices and transaction fees set by the DSO.

\section{A. Day-Ahead Prosumer Preferences}

In the day-ahead P2P market, the prosumers are connected by a set of potential bilateral energy contracts $X^{F}$. Each contract $x \in X^{F}$ specifies the transfer of a discrete quantity of energy $\Delta q$ during a particular trading interval $t_{x} \in \mathcal{T}$ from a seller $s_{x} \in \mathcal{P}$ to a buyer $b_{x} \in \mathcal{P}$. Each bilateral contract has a buyer price $\lambda_{x}^{b}$ and a seller price $\lambda_{x}^{s}$. For prosumer $i, X_{\rightarrow i}^{F}=\left\{x \in X^{F} \mid b_{x}=i\right\}$ is the set of available upstream contracts, and $X_{i \rightarrow}^{F}=\left\{x \in X^{F} \mid s_{x}=i\right\}$ is the set of available downstream contacts. These can be further divided into contracts associated with particular intervals, $X_{\rightarrow i t}^{F}=\left\{x \in X_{\rightarrow i}^{F} \mid t_{x}=t\right\}, X_{i \rightarrow t}^{F}=\left\{x \in X_{i \rightarrow}^{F} \mid t_{x}=t\right\}$.

The preferences of prosumer $i \in \mathcal{P}$ in the day-ahead $\mathrm{P} 2 \mathrm{P}$ market can be formulated as a mixed-integer linear program,

$\max \sum_{t \in \mathcal{T}}\left(\lambda_{i t}^{e x p} q_{i t}^{e x p}-\lambda_{i t}^{i m p} q_{i t}^{i m p}\right)+\sum_{x \in X_{i \rightarrow}^{F}}\left(\lambda_{x}^{s}-\frac{\delta_{i b_{x} t_{x}}}{2}\right) q_{i x}$

$-\sum_{x \in X_{\rightarrow i}^{F}}\left(\lambda_{x}^{b}+\frac{\delta_{s_{x} i t_{x}}}{2}\right) q_{i x}-\Delta t c_{i}^{d e g}\left(p_{i t}^{c h}+p_{i t}^{d i s}\right)$

s.t. $\Delta t\left(\hat{p}_{i t}^{\text {load }}-p_{i t}^{p v}+p_{i t}^{c h}-p_{i t}^{d i s}\right)$

$=q_{i t}^{i m p}-q_{i t}^{e x p}+\sum_{x \in X_{\rightarrow i t}^{F}} q_{i x}-\sum_{x \in X_{i \rightarrow t}^{F}} q_{i x}$,

$q_{i x} \in\{0, \Delta q\}, 0 \leq q_{i t}^{e x p}, 0 \leq q_{i t}^{i m p}$,

(1b), (1c), (1d).

The decision variables are the energy storage system charging and discharging powers $p_{i t}^{c h}, p_{i t}^{d i s}, t \in \mathcal{T}$, the PV generation $p_{i t}^{p v}, t \in \mathcal{T}$, the energy quantities planned to be bought from and sold to the DSO $q_{i t}^{i m p}, q_{i t}^{\text {exp }}, t \in \mathcal{T}$ and energy quantities associated with the potential P2P contracts $q_{i x}, x \in X_{\rightarrow i}^{F} \cup$ $X_{i \rightarrow}^{F} \cdot q_{i x}^{*}=0$ indicates a rejected contract, while $q_{i x}^{*}=\Delta q$ indicates an accepted contract. For a downstream contract $x \in$ $X_{i \rightarrow}^{F}, \delta_{i b_{x} t_{x}}$ is the associated $\mathrm{P} 2 \mathrm{P}$ transaction fee from (8) between agent $i$ and the buyer $b_{x}$. Similarly, for an upstream contract $x \in X_{\rightarrow i}^{F}, \delta_{s_{x} i t_{x}}$ is the P2P transaction fee between the seller $s_{x}$ and agent $i$.

The objective (9a) is to maximise the net revenue from energy trading with the DSO and other prosumers, less the cost of battery degradation. (9b) is a power balance constraint and (9c) puts limits on the energy quantity decision variables. (1b)-(1d), are relevant individual prosumer constraints from the day-ahead OPF problem.

\section{B. Intra-Day Prosumer Preferences}

Intra-day $\mathrm{P} 2 \mathrm{P}$ markets are organised for each trading interval. $X_{t}^{R}$ is the set of potential bilateral energy contracts for the intra-day market for interval $t$. In the intra-day markets, the prosumers have the opportunity to engage in $\mathrm{P} 2 \mathrm{P}$ trading and adjust their PV sources based on updated predictions of their load $p_{i t}^{\text {load }}$ and available renewable generation $\bar{p}_{i t}^{p v}$.
The preferences of prosumer $i \in \mathcal{P}$ in the intra-day $\mathrm{P} 2 \mathrm{P}$ market for interval $t$ can be formulated as a mixed-integer linear program,

$$
\begin{aligned}
\max \quad & \lambda_{i t}^{e x p} q_{i t}^{e x p}-\lambda_{i t}^{i m p} q_{i t}^{i m p}+\sum_{x \in X_{i \rightarrow t}^{R}}\left(\lambda_{x}^{s}-\frac{\delta_{i b_{x} t}}{2}\right) q_{i x} \\
& -\sum_{x \in X_{\rightarrow i t}^{R}}\left(\lambda_{x}^{b}+\frac{\delta_{s_{x} i t}}{2}\right) q_{i x} \\
\text { s.t. } \Delta & t\left(p_{i t}^{\text {load }}-p_{i t}^{p v}+p_{i t}^{c h *}-p_{i t}^{d i s *}\right) \\
= & q_{i t}^{i m p}-q_{i t}^{e x p}+\sum_{x \in X_{\rightarrow i t}^{R}} q_{i x}-\sum_{x \in X_{i \rightarrow t}^{R}} q_{i x} \\
& +\sum_{x \in X_{\rightarrow i t}^{F}} q_{i x}^{*}-\sum_{x \in X_{i \rightarrow t}^{F}} q_{i x}^{*} \\
q_{i x} & \in\{0, \Delta q\}, 0 \leq q_{i t}^{e x p}, 0 \leq q_{i t}^{i m p} \\
0 \leq & p_{i t}^{p v} \leq \bar{p}_{i t}^{p v} .
\end{aligned}
$$

The decision variables are the PV generation $p_{i t}^{p v}$, the energy quantities bought from and sold to the DSO $q_{i t}^{i m p}, q_{i t}^{\text {exp }}$ and the energy quantities associated with the available P2P contracts $q_{i x}, x \in X_{\rightarrow i t}^{R} \cup X_{i \rightarrow t}^{R}$. It is assumed that the prosumers keep their energy storage system schedules decided during the day-ahead multi-period P2P market, $p_{i t}^{c h *}, p_{i t}^{d i s *}$. The contract energy quantities agreed in the day-ahead market are given by $q_{x}^{*}, x \in X_{\rightarrow i t}^{F} \cup X_{i \rightarrow t}^{F}$.

\section{P2P Negotiation}

Algorithm 1 shows the iterative P2P negotiation mechanism from [18] which the prosumers use to reach agreement on energy contract quantities and prices in the day-ahead and intra-day P2P markets. The mechanism is based on a deferred acceptance algorithm for networked matching markets [50].

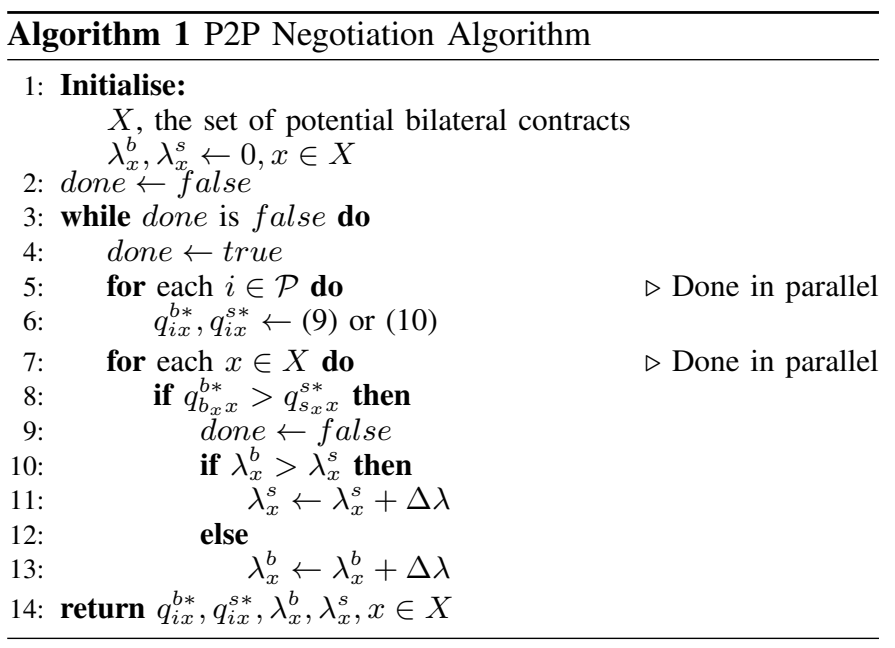

The negotiation mechanism allows prosumers to make autonomous utility-maximising decisions, and is fully distributed, since each contract's price is adjusted based only on the decisions of its buyer and seller. The granularity of the market price increment $\Delta \lambda$, contract energy unit $\Delta q$ and trading interval duration $\Delta t$ offers a trade-off: more granularity creates additional opportunities for mutually beneficial $\mathrm{P} 2 \mathrm{P}$ transactions, but increases the negotiation time. The negotiation mechanism is guaranteed to finish within a finite number of iterations. This can be seen by considering that: contract prices always increase; prior to completion at least one contract will have its prices increased at each iteration; and 


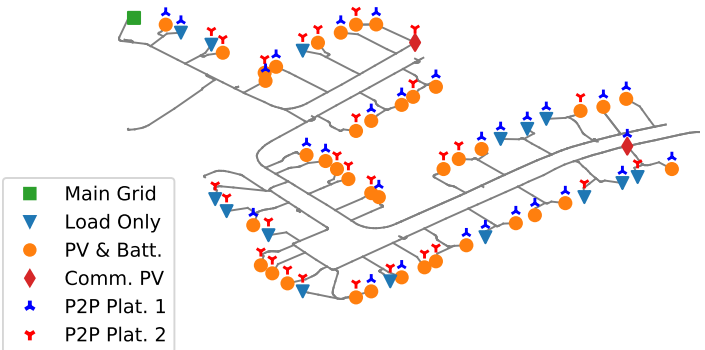

Fig. 2: The case study distribution network, with 57 prosumers ( 15 with singlephase inflexible loads, 40 with single-phase inflexible loads, PV sources and battery energy storage systems and 2 three-phase community-scale PV plants). The prosumers are divided between two $\mathrm{P} 2 \mathrm{P}$ trading platforms.

that prosumers will reject all upstream contracts with buyer prices greater than the DSO import price for that interval.

Note that in the case where prosumers operate under the DSO pricing scheme without being part of a local P2P platform, their decision making is captured by (9) and (10) with $X^{F}, X_{t}^{R}=\varnothing$.

\section{RESULts}

Simulation case studies are presented to demonstrate the operation of the proposed market design. The design is implemented for the IEEE European Low Voltage Test Feeder, shown in Fig. 2 [51], with 57 prosumers. 55 are residential prosumers with single-phase connections. Of these, 40 have PV sources $(4 \mathrm{kWp})$ and batteries $(4 \mathrm{~kW}, 8 \mathrm{kWh})$, and 15 only have inflexible loads. There are also 2 community-scale PV plant prosumers $(60 \mathrm{kWp})$ with three-phase connections.

Residential load and PV generation data, with 30 minute resolution, is obtained from the Customer-Led Network Revolution Trial from June 2014 [52]. This data is used to develop the probability distributions the DSO uses for the point estimate method. The averages of the load and renewable generation over the month are used for the prosumer predictions for day-ahead trading. It is assumed that the phase voltages at the grid connection point are balanced with magnitude of $1.00 \mathrm{pu}$, and that the network has phase voltage magnitude limits of 0.95 pu to 1.05 pu [53]. The upstream energy prices at each 30-minute time interval are assumed to follow normal distributions, with mean values that vary between $£ 0.1 / \mathrm{kWh}$ and $£ 0.2 / \mathrm{kWh}$ (in proportion with the monthly average load). The upstream energy price has a standard deviation which is equal to $25 \%$ of the mean value for each time interval. For the PV generation, the maximum standard deviation occurs during the time interval in the middle of the day, and is $30 \%$ of the rated power. For the individual household loads, the standard deviation is $47 \%$ of the daily mean value. It is assumed that the prosumer batteries start the day at 50\% charge and must end the day with the same charge, and have degradation costs $c_{i}^{d e g}=£ 0.05 / \mathrm{kWh}$. Note that for the case studies battery charging and discharging losses have not been considered.

The prosumers are divided between two P2P platforms and can negotiate bilateral energy transactions with other prosumers that are part of the same platform. The P2P trading platforms have trading intervals $\Delta t=30$ minutes, contract

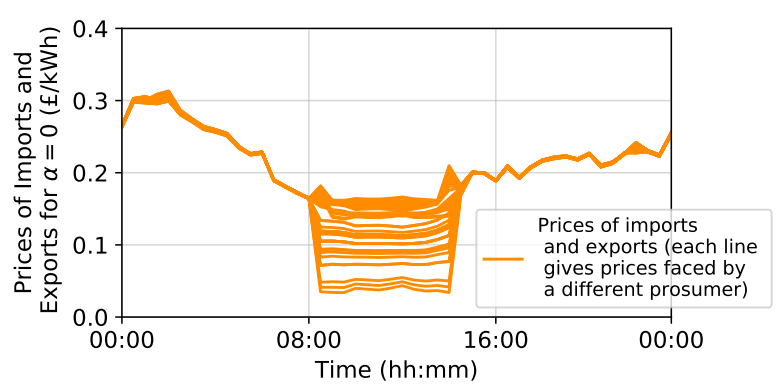

(a)

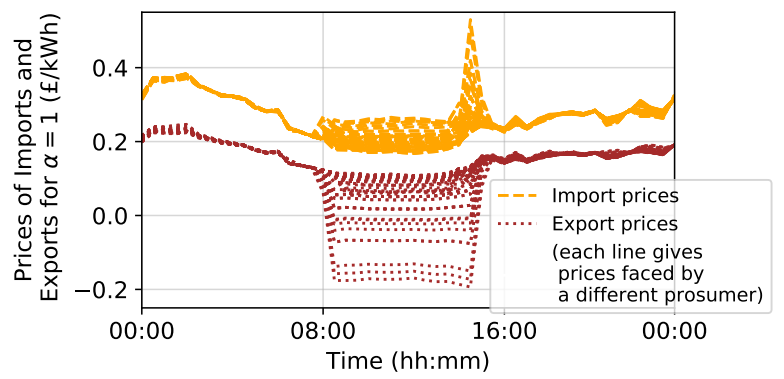

(b)

Fig. 3: The equivalent locational import and export prices faced by the prosumers over the day. Each line represents the prices faced by a different prosumer. (a) For a price-spread scale factor of 0 . In this case, the prices of imports and exports are the same. (b) For a price-spread scale factor of 1 .

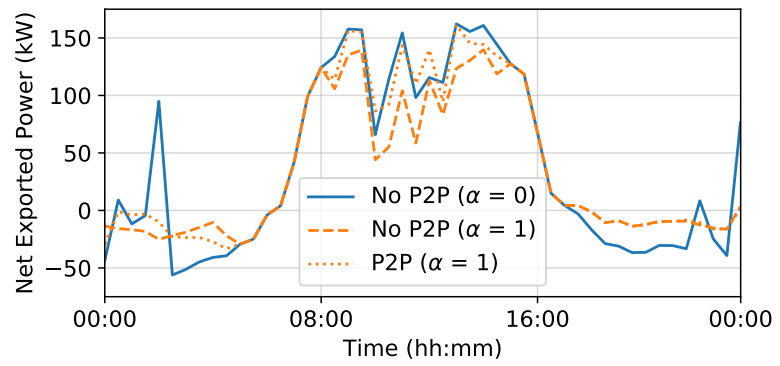

Fig. 4: The net power exported to the main grid when the price-spread scale factor $\alpha$ is 0 without P2P energy trading, and when the scale factor is 1 , with and without $\mathrm{P} 2 \mathrm{P}$ energy trading.

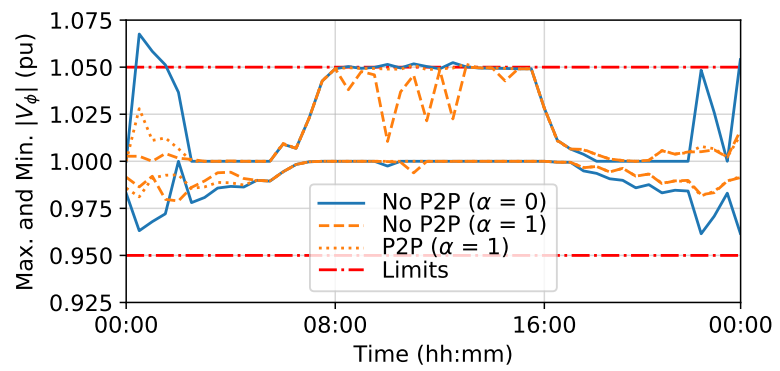

Fig. 5: The maximum and minimum phase voltage magnitudes across the network when the price-spread scale factor $\alpha$ is 0 without $\mathrm{P} 2 \mathrm{P}$ energy trading, and when the scale factor is 1 , with and without $\mathrm{P} 2 \mathrm{P}$ energy trading.

energy units $\Delta q=0.5 \mathrm{kWh}$ and price increments $\Delta \lambda=$ $£ 0.05 / \mathrm{kWh}$.

The DSO uses the day-ahead OPF problem to estimate the probabilistic DLMPs and then calculates unidirectional locational import and export prices and P2P transaction fees. 


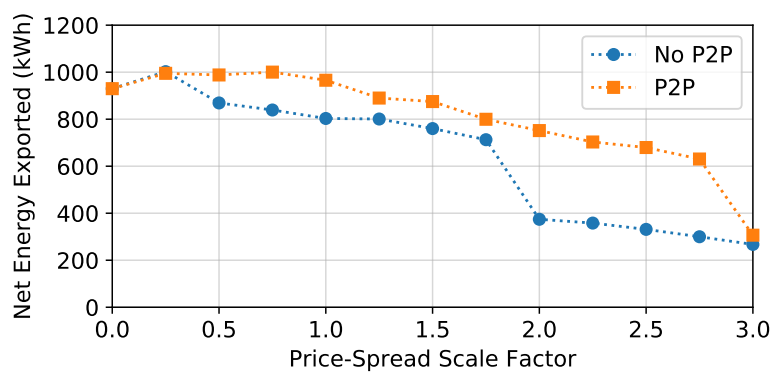

Fig. 6: The net energy exported for different price-spread scale factors, with and without $\mathrm{P} 2 \mathrm{P}$ energy trading.

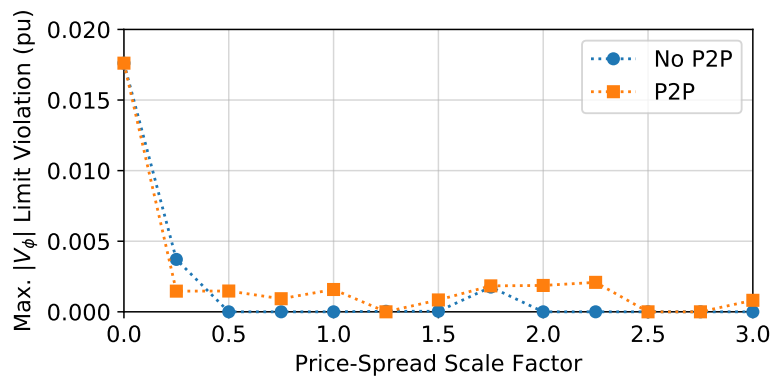

Fig. 7: The maximum absolute voltage limit violations for different pricespread scale factors, with and without P2P energy trading. Note that for the case studies, all violations are of the upper voltage limit.

Fig. 3a shows the mean DLMPs for the phases the prosumers are connected to over the day. These are used as import and export prices when the price-spread scale factor $\alpha=0$. Fig $3 \mathrm{~b}$ shows the import and export prices when the scale factor is 1 (the mean DLMPs plus and minus one standard deviation). The DLMPs diverge during the middle of the day when the export on certain buses is constrained by the network voltage limits. It is assumed that the DSO can directly communicate with and curtail the community-scale PV plants during operation if necessary to enforce the network constraints (by solving an intra-day version of (1)). However, in this case the DSO must remunerate the PV plants as if they had not been curtailed, which negatively impacts DSO revenue.

Fig. 4 shows the net power exported to the main grid for three case studies: (i) with a price-spread scale factor of 0 , without P2P trading; (ii) with a scale factor of 1 , without P2P trading; and (iii) with a scale factor of 1 , with P2P trading. Fig. 5 shows the maximum and minimum phase voltage magnitudes across the network for each case. Note that when the scale factor is 0 , there is no price-spread and P2P energy trading does not affect the exported power and voltage profiles (although it does affect the relative revenues of the DSO and the prosumers).

When the price-spread scale factor is 0 , the prosumers are exposed to the DLMPs in Fig. 3a. The prosumers are incentivised to discharge their batteries when the price of energy is highest, which occurs around 1 am for the particular price profile in this case, and they are incentivised to charge their batteries when the price of energy is low during the middle of the day. The DSO also curtails the communityscale PV plants during the day as required to prevent the

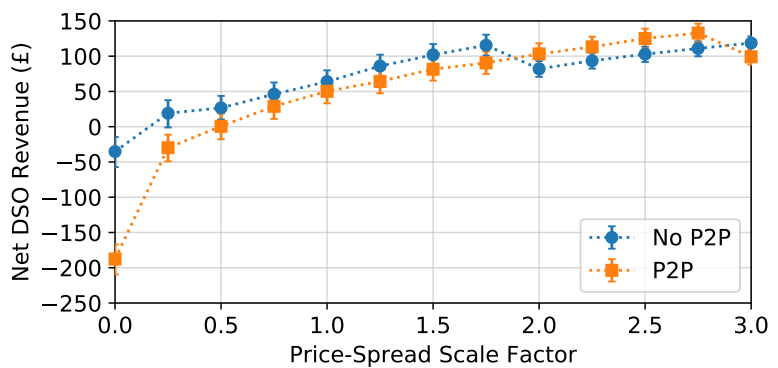

Fig. 8: The net DSO revenue for different price-spread scale factors, with and without $\mathrm{P} 2 \mathrm{P}$ energy trading. The error bars show the variation given the upstream energy price variability ( $90 \%$ confidence intervals).

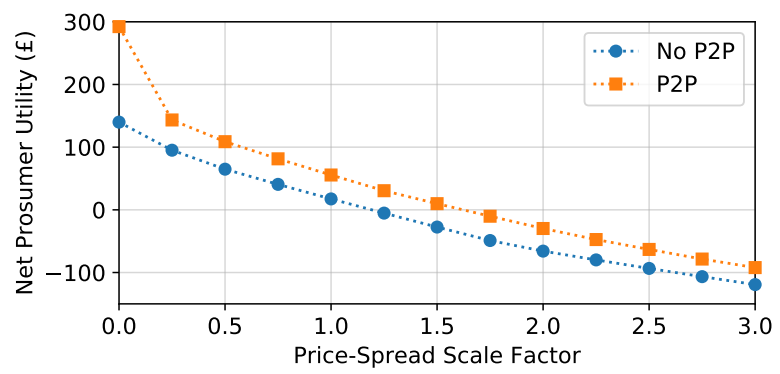

Fig. 9: The total net prosumer utility (net revenue less degradation costs) for different price-spread scale factors, with and without P2P energy trading.

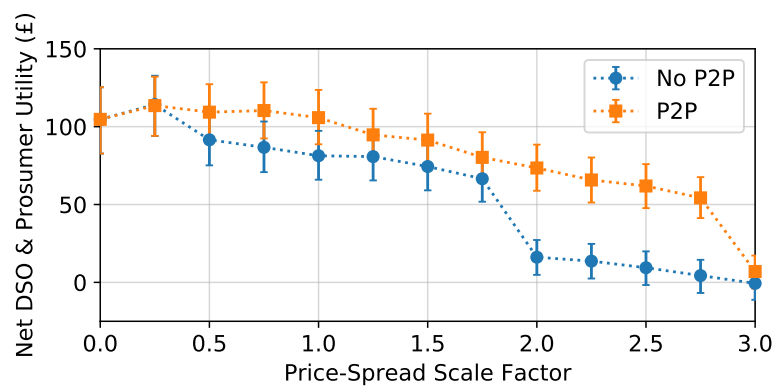

Fig. 10: The total net DSO and prosumer utility for different price-spread scale factors. The error bars show the variation given the upstream energy price variability ( $90 \%$ confidence intervals).

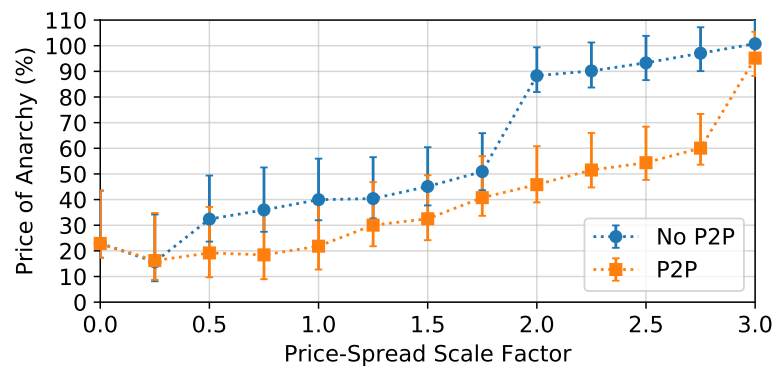

Fig. 11: The price of anarchy for different price-spread scale factors, with and without $\mathrm{P} 2 \mathrm{P}$ energy trading. The error bars show the variation given the upstream energy price variability ( $90 \%$ confidence intervals).

maximum voltage limit from being violated. However, the upper voltage limits are still violated around $1 \mathrm{am}$, due to multiple prosumers discharging their batteries. The voltage violations are addressed with the scale factor increased to 1 , since the prosumers are incentivised to use their batteries to 
increase their individual self-consumption. However, without P2P energy trading, the price-spread causes individual prosumers to excessively curtail their renewable generation during the day, resulting in underutilisation of the available network capacity. Introducing P2P energy trading allows prosumers to organise mutually beneficial local transactions, reducing renewable curtailment.

Fig. 6 shows the net exported energy over the day and Fig. 7 show the maximum voltage limit violations as the price-spread scale factor is increased, with and without P2P trading. Fig. 8 and Fig. 9 show the net DSO revenue and the net utility of the prosumers. Note that DSO revenue includes upstream trading, trading with prosumers and $\mathrm{P} 2 \mathrm{P}$ transaction fees. Fig. 8 includes error bars showing the revenue variation given the upstream energy price variability ( $90 \%$ confidence intervals). Prosumer utility includes energy trading with the DSO, P2P trading, $\mathrm{P} 2 \mathrm{P}$ transaction fees and battery degradation costs. Fig. 10 shows the total net utility, which is equal to the sum of the DSO revenue and the net utility of the prosumers.

To understand the benefits of the proposed market design, first consider the case when the price-spread scale factor is zero. This corresponds to a standard unidirectional pricing strategy using DLMPs. From Fig. 8, it can be seen that the DSO consistently has negative net revenue, which will not be sustainable. Also, from Fig. 7, it can be seen that there are voltage limit violations. Increasing the scale factor from 0 to 0.25 increases the average overall net utility by $9.2 \%$, due to a reduction in renewable curtailment, which can be seen in Fig. 6 as an increase in net exported energy. From Fig. 7 it can be seen that the worst-case voltage limit violations are also significantly reduced. However, as shown in Fig. 10, when $\mathrm{P} 2 \mathrm{P}$ trading is not enabled, increasing the scale factor beyond 0.25 reduces overall utility, since it incentivises conservative DER utilisation. Enabling P2P trading increases overall utility by reducing the curtailment of renewable generation. Without P2P trading, increasing the scale factor from 0.25 to 1 results in an average reduction in net utility of $28.9 \%$, whereas with $\mathrm{P} 2 \mathrm{P}$ trading the reduction is only $7.4 \%$.

Fig. 8 shows that P2P energy trading reduces the DSO's revenue when the price-spread scale factor is below 2. Without P2P trading, battery owners are only incentivised to buy energy from the DSO for storage if it lets them reduce their own energy demand during time intervals with higher prices. When $\mathrm{P} 2 \mathrm{P}$ energy trading is enabled, battery owners can buy energy from the DSO for storage at intervals with low prices, and then sell it to other prosumers during intervals with higher prices. This reduces the DSO's revenue, with the effect being most pronounced when the price-spread scale factor is zero.

Fig. 10 shows that without P2P trading, a significant reduction in utility occurs when the price-spread scale factor increases from 1.75 to 2 . This is caused by the export price at one of the community-scale PV plants becoming negative during an interval with significant generation, causing a step increase in curtailment. With $\mathrm{P} 2 \mathrm{P}$ trading, it remains profitable for this energy to be traded to prosumers with batteries for later export, until the scale factor increases from 2.75 to 3 . When the price-spread scale factor is between 2 and 2.75, P2P trading increases the DSO's revenue, since it enables a significant amount of PV generation to be traded locally, rather than curtailed, and the P2P transaction fees are relatively high.

To calculate a price of anarchy under the proposed market design, the centralised day-ahead dispatch problem (1) was solved using expected values for the random variables. Then, the price of anarchy was calculated as the percentage reduction in overall utility under the proposed market design compared with the centralised dispatch solution. Fig. 11, shows the price of anarchy for different price-spread scale factors.

The negotiation time for day-ahead and intra-day P2P trading depends on the number of iterations required for Algorithm 1 to converge, and the iteration time. With the prosumer decision problems ((9) or (10)) solved in parallel, the iteration time is given by the slowest prosumer decision problem solution time, plus communication overheads. The case studies were completed on a dual-core $1.6 \mathrm{GHz}$ Intel Core i5 CPU with $8 \mathrm{~GB}$ of RAM, using a serial implementation. The decision problem solution times have been tracked to calculate the computation time which would be required for a parallel implementation. Note that practical implementation requires intra-day negotiation to be completed within the 30-minute trading interval duration. The number of iterations required to reach convergence increases with the price-spread scale factor, while the individual decision problem solution times did not vary significantly. For a price-spread scale factor of 0.5 , day-ahead P2P trading took 124 iterations, with a worst-case iteration time of $5.96 \mathrm{~s}$. Excluding communication overheads, this would give a parallel computation time of $12.3 \mathrm{~min}$. Intraday P2P trading took up to 104 iterations, with a worst-case iteration time of $1.78 \mathrm{~s}$, giving a parallel computation time of $3.1 \mathrm{~min}$. The longest computation times occurred for a price-spread scale factor of 3 . In this case, day-ahead trading required 366 iterations, with a worst-case iteration time of $6.01 \mathrm{~s}$, giving a parallel computation time of $36.7 \mathrm{~min}$. Intraday trading required up to 744 iterations, with a worst-case iteration time of $1.78 \mathrm{~s}$, giving a parallel computation time of $22.1 \mathrm{~min}$.

\section{CONCLUSION}

This paper has presented a new local energy market design for distribution systems, combining unidirectional pricing based on probabilistic DLMPs and local P2P energy trading platforms. The unidirectional pricing scheme provides a scalable strategy for a DSO to incentivise coordination between prosumer-owned DERs, accounting for network constraints and sources of uncertainty. It has been shown that integrating local P2P energy trading platforms can create value for prosumers, and the system as a whole, by reducing the curtailment of renewable generation.

An important area for future work would be to design a strategy allowing a regulator to select a price-spread scale factor which best balances their particular objectives for the market. The aim would be to select a price-spread scale factor which achieves a desired trade-off between overall economic efficiency, DSO profits and the probability of network constraint violations. A promising approach could be to formulate this as a bi-level decision problem, with the regulator's preferences defining the upper-level objective. There are likely to be 
challenges to overcome, particularly in terms of computational complexity and predicting how the market will respond to different scale factors. There is also the potential for the scale factor to be updated and refined over time in response to new information.

Another area for future work is to model the upstream market in more detail. In this paper, only 30-minute energy trading has been considered. However, there is also the potential to allow prosumers to provide upstream ancillary services, such as frequency response and voltage support. Also, it has been assumed that the local market is small relative to the wholesale market, so that local demand does not directly impact upstream energy prices. However, this may not be a good assumption if DSO operated local markets become widespread and provide a significant share of overall generation and flexibility. One approach for managing this would be to include DSOs within wholesale market bidding, with DSOs responsible for buying/selling the net energy imports/exports of the local markets they manage.

\section{APPENDIX}

Let $X=\left(X_{1}, \ldots, X_{n}\right)$ be the vector of random variables, $\left(X_{1}, \ldots, X_{n}\right)=\left(\hat{\lambda}_{01}, \ldots, \hat{\lambda}_{0 T}, \hat{x}_{1}^{p v}, \ldots, \hat{x}_{T}^{p v}, \hat{x}_{1}^{\text {load }}, \ldots, \hat{x}_{P}^{\text {load }}\right)$ $X$ has mean vector $\mu_{X}$, covariance matrix $C_{X}$, skewness vector $\gamma_{X}$ and kurtosis vector $\kappa_{X}$, Using the Cholesky decomposition $C_{X}=L L^{\top}$, the correlated random variables in $X$ can be transformed into uncorrelated ones $X^{\prime}$, with statistical moments given by [46]

$$
\begin{aligned}
\mu_{X^{\prime}} & =L^{-1} \mu_{X}, C_{X^{\prime}}=L^{-1} C_{X}\left(L^{-1}\right)^{\top}, \\
\gamma_{X_{i}^{\prime}} & =\sum_{j=1}^{n}\left(L_{i, j}^{-1}\right)^{3} \gamma_{X_{j}} \sigma_{X_{j}}^{3}, \\
\kappa_{X_{i}^{\prime}} & =\sum_{j=1}^{n}\left(L_{i, j}^{-1}\right)^{4} \kappa_{X_{j}} \sigma_{X_{j}}^{4} .
\end{aligned}
$$

$L_{i, j}^{-1}$ is the element at the $i$ th row and $j$ th column of $L^{-1}$.

For each $X_{i}^{\prime}$, uncorrelated estimate points $x_{0}^{\prime}, x_{i-}^{\prime}, x_{i+}^{\prime}$, and associated weights $w_{0}, w_{i-}, w_{i+}$, are given by [45]

$$
\begin{aligned}
x_{i 0}^{\prime} & =\mu_{X_{i}^{\prime}}, \quad w_{0}=1-w_{i+}-w_{i-} \\
x_{i+}^{\prime} & =\mu_{X_{i}^{\prime}}+\xi_{i+} \sigma_{X_{i}^{\prime}}, w_{i+}=\frac{1}{x_{i+}^{\prime}\left(x_{i+}^{\prime}+x_{i-}^{\prime}\right)}, \\
x_{i-}^{\prime} & =\mu_{X_{i}^{\prime}}+\xi_{i-} \sigma_{X_{i}^{\prime}}, w_{i-}=\frac{1}{x_{i-}^{\prime}\left(x_{i+}^{\prime}+x_{i-}^{\prime}\right)}, \\
\xi_{i+} & =\frac{\gamma_{X_{i}^{\prime}}}{2}+\sqrt{\kappa_{X_{i}^{\prime}}-\frac{3 \gamma_{X_{i}^{\prime}}^{2}}{4}}, \xi_{i-}=-\frac{\gamma_{X_{i}^{\prime}}}{2}+\sqrt{\kappa_{X_{i}^{\prime}}-\frac{3 \gamma_{X_{i}^{\prime}}^{2}}{4}} .
\end{aligned}
$$

The $2 n+1$ uncorrelated estimate vectors are given by

$$
\begin{aligned}
& X_{0}^{\prime}=\left(x_{10}^{\prime}, \ldots, x_{n 0}^{\prime}\right), \\
& X_{i+}^{\prime}=\left(x_{10}^{\prime}, \ldots, x_{i+}^{\prime}, \ldots, x_{n 0}^{\prime}\right), i \in\{1, \ldots, n\}, \\
& X_{i-}^{\prime}=\left(x_{10}^{\prime}, \ldots, x_{i-}^{\prime}, \ldots, x_{n 0}^{\prime}\right), i \in\{1, \ldots, n\} .
\end{aligned}
$$

The associated correlated estimate vectors are obtained by applying $X=B^{-1} X^{\prime}$ to (17)-(19). Then, DLMPs and differential DLMPs are calculated by solving (1) for each of these. For a given output $y_{j}$ with values for the estimate vectors of $y_{j 0}, y_{j i+}, y_{j i-}, i \in\{1, \ldots, n\}$, the mean $\mu_{y_{j}}$ and variance $\sigma_{y_{j}}^{2}$ are estimated as [45]

$$
\mu_{y_{j}}=\left(1-\sum_{i=1}^{n}\left(w_{i+}+w_{i-}\right)\right) y_{j 0}
$$

$$
\begin{aligned}
& +\sum_{i=1}^{n}\left(w_{i+} y_{j i+}+w_{i-} y_{j i-}\right) \\
\sigma_{y_{j}}^{2} & =\sum_{i=1}^{n}\left(w_{i+}\left(y_{j i+}-y_{j 0}\right)^{2}+w_{i-}\left(y_{j i-}-y_{j 0}\right)^{2}\right. \\
& \left.-\left(w_{i+}+w_{i-}\right)\left(w_{i+}\left(y_{j i+}-y_{j 0}\right)+w_{i-}\left(y_{j i-}-y_{j 0}\right)\right)^{2}\right) .
\end{aligned}
$$

\section{REFERENCES}

[1] Y. Parag and B. K. Sovacool, "Electricity Market Design for the Prosumer Era," Nature Energy, vol. 1, no. 4, p. 16032, 2016.

[2] D. Pudjianto, et al., "Value of Integrating Distributed Energy Resources in the UK Electricity System," IEEE PES General Meeting, pp. 1-6, 2010.

[3] C. Defeuilley, "Retail Competition in Electricity Markets," Energy Policy, vol. 37, no. 2, pp. 377-386, 2009.

[4] C. Eid, et al., "Managing Electric Flexibility from Distributed Energy Resources: A Review of Incentives for Market Design," Renewable and Sustainable Energy Reviews, vol. 64, pp. 237-247, 2016.

[5] M. N. Faqiry, et al., "A Day-Ahead Market Energy Auction for Distribution System Operation," IEEE International Conference on Electro Information Technology, pp. 182-187, 2017.

[6] F. Lezama, et al., "Local Energy Markets: Paving the Path Towards Fully Transactive Energy Systems," IEEE Transactions on Power Systems, 2019 (Early Access).

[7] S. Gill, I. Kockar, and G. W. Ault, "Dynamic Optimal Power Flow for Active Distribution Networks," IEEE Transactions on Power Systems, vol. 29, no. 1, pp. 121-131, 2014.

[8] T. Morstyn, B. Hredzak, R. P. Aguilera, and V. G. Agelidis, "Model Predictive Control for Distributed Microgrid Battery Energy Storage Systems," IEEE Transactions on Control Systems Technology, vol. 26, no. 3, pp. 1107-1114, 2018.

[9] W. Tushar, W. Saad, H. V. Poor, and D. B. Smith, "Economics of Electric Vehicle Charging: A Game Theoretic Approach," IEEE Transactions on Smart Grid, vol. 3, no. 4, pp. 1767-1778, 2012.

[10] A. Mondal, S. Misra, and M. S. Obaidat, "Distributed Home Energy Management System With Storage in Smart Grid Using Game Theory," IEEE Systems Journal, vol. 11, no. 3, pp. 1857-1866, 2017.

[11] Z. Zhu, S. Lambotharan, W. H. Chin, and Z. Fan, "A Mean Field Game Theoretic Approach to Electric Vehicles Charging," IEEE Access, vol. 4, pp. 3501-3510, 2016.

[12] A. De Paola, D. Angeli, and G. Strbac, "Price-Based Schemes for Distributed Coordination of Flexible Demand in the Electricity Market," IEEE Transactions on Smart Grid, vol. 8, no. 6, pp. 3104-3116, 2017.

[13] E. Loukarakis, C. J. Dent, and J. W. Bialek, "Decentralized Multi-Period Economic Dispatch for Real-Time Flexible Demand Management,' IEEE Transactions on Power Systems, vol. 31, no. 1, pp. 672-684, 2016.

[14] T. Morstyn, B. Hredzak, and V. G. Agelidis, "Network Topology Independent Multi-Agent Dynamic Optimal Power Flow for Microgrids With Distributed Energy Storage Systems," IEEE Transactions on Smart Grid, vol. 9, no. 4, pp. 3419-3429, 2018.

[15] C. Edmunds, W. A. Bukhsh, and S. Galloway, "The Impact of Distribution Locational Marginal Prices on Distributed Energy Resources: An Aggregated Approach," International Conference on the European Energy Market, 2018.

[16] L. Cheng, et al., "Distribution Locational Marginal Pricing for Optimal Electric Vehicle Charging Through Chance Constrained Mixed-Integer Programming," IEEE Transactions on Smart Grid, vol. 9, no. 2, pp. 644-654, 2016

[17] L. Bai, et al., "Distribution Locational Marginal Pricing (DLMP) for Congestion Management and Voltage Support," IEEE Transactions on Power Systems, vol. 33, no. 4, pp. 4061-4073, 2018.

[18] T. Morstyn, A. Teytelboym, and M. D. McCulloch, "Bilateral Contract Networks for Peer-to-Peer Energy Trading," IEEE Transactions on Smart Grid, vol. 10, no. 2, pp. 2026-2035, 2019.

[19] T. Morstyn, A. Teytelboym, and M. D. McCulloch, "Matching Markets with Contracts for Electric Vehicle Smart Charging," in IEEE Power \& Energy Society General Meeting (PESGM), 2018, pp. 1-5.

[20] T. Morstyn and M. McCulloch, "Multi-Class Energy Management for Peer-to-Peer Energy Trading Driven by Prosumer Preferences," IEEE Transactions on Power Systems, 2018 (Early Access).

[21] T. Baroche, P. Pinson, R. Le Goff Latimier, and H. Ben Ahmed, "Exogenous Cost Allocation in Peer-to-Peer Electricity Markets," IEEE Transactions on Power Systems, 2019 (Early Access).

[22] J. Guerrero, A. C. Chapman, and G. Verbic, "Decentralized P2P Energy Trading under Network Constraints in a Low-Voltage Network," IEEE Transactions on Smart Grid, 2018 (Early Access). 
[23] J. Kim and Y. Dvorkin, "Peer-to-Peer Energy Trading in Distribution Systems," Computing Research Repository, vol. abs/1902.03940, 2019.

[24] S. Wang, A. F. Taha, and J. Wang, "Blockchain-Assisted Crowdsourced Energy Systems," in IEEE Power \& Energy Society General Meeting (PESGM), 2018, pp. 1-5.

[25] S. Wang, et al., "Energy Crowdsourcing and Peer-to-Peer Energy Trading in Blockchain-Enabled Smart Grids," IEEE Transactions on Systems, Man, and Cybernetics: Systems, vol. 49, no. 8, pp. 1612-1623, 2019.

[26] J. Kang, et al., "Enabling Localized Peer-to-Peer Electricity Trading among Plug-in Hybrid Electric Vehicles Using Consortium Blockchains," IEEE Transactions on Industrial Informatics, vol. 13, no. 6, pp. 3154-3164, 2017.

[27] A. Paudel, K. Chaudhari, C. Long, and H. B. Gooi, "Peer-to-peer Energy Trading in a Prosumer-Based Community Microgrid: A Game-Theoretic Model," IEEE Transactions on Industrial Electronics, vol. 66, no. 8, pp. 6087-6097, 2019.

[28] A. Papavasiliou, "Analysis of Distribution Locational Marginal Prices," IEEE Transactions on Smart Grid, vol. 9, no. 5, pp. 4872-4882, 2018.

[29] S. Bose and S. H. Low, "Some Emerging Challenges in Electricity Markets," in Smart Grid Control: Overview and Research Opportunities, 2019, pp. 29-45.

[30] C. Long, J. Wu, Y. Zhou, and N. Jenkins, "Peer-to-Peer Energy Sharing Through a Two-Stage Aggregated Battery Control in a Community Microgrid," Applied Energy, vol. 226, pp. 261-276, 2018.

[31] L. Han, T. Morstyn, and M. McCulloch, "Constructing Prosumer Coalitions for Energy Cost Savings Using Cooperative Game Theory," in Power Systems Computation Conference (PSCC), 2018, pp. 1-7.

[32] L. Han, T. Morstyn, and M. McCulloch, "Incentivizing Prosumer Coalitions With Energy Management Using Cooperative Game Theory," IEEE Transactions on Power Systems, vol. 34, no. 1, pp. 303-313, 2019.

[33] S. Boyd, et al., "Distributed Optimization and Statistical Learning via the Alternating Direction Method of Multipliers," Foundations and Trends in Machine Learning, vol. 3, no. 1, pp. 1-122, 2011.

[34] M. Kraning, E. Chu, J. Lavaei, and S. Boyd, "Dynamic Network Energy Management via Proximal Message Passing," Foundations and Trends in Optimization, vol. 1, no. 2, pp. 70-122, 2014.

[35] L. Kristov, P. De Martini, and J. D. Taft, "A Tale of Two Visions: Designing a Decentralized Transactive Electric System," IEEE Power and Energy Magazine, vol. 14, no. 3, pp. 63-69, 2016.

[36] R. Li, Q. Wu, and S. S. Oren, "Closure to Discussion on "Distribution Locational Marginal Pricing for Optimal Electric Vehicle Charging Management"," IEEE Transactions on Power Systems, vol. 29, no. 4, pp. 1867-1867, 2014

[37] T. Morstyn, N. Farrell, S. J. Darby, and M. D. McCulloch, "Using Peer-to-Peer Energy-Trading Platforms to Incentivize Prosumers to Form Federated Power Plants," Nature Energy, vol. 3, no. 2, pp. 94-101, 2018.

[38] T. Morstyn, A. Teytelboym, and M. D. McCulloch, "Designing Decentralized Markets for Distribution System Flexibility," IEEE Transactions on Power Systems, vol. 34, no. 3, pp. 2128-2139, 2019.

[39] J. Ossenbrink, "How Feed-In Remuneration Design Shapes Residential PV Prosumer Paradigms," Energy Policy, vol. 108, pp. 239-255, 2017.

[40] T. Morstyn, B. Hredzak, and V. G. Agelidis, "Control Strategies for Microgrids With Distributed Energy Storage Systems: An Overview," IEEE Transactions on Smart Grid, vol. 9, no. 4, pp. 3652-3666, 2018.

[41] A. Bernstein and E. Dall'anese, "Linear Power-Flow Models in Multiphase Distribution Networks," IEEE PES Innovative Smart Grid Technologies Conference Europe, pp. 1-6, 2017.

[42] Z. Ma, D. S. Callaway, and I. A. Hiskens, "Decentralized Charging Control of Large Populations of Plug-in Electric Vehicles," IEEE Transactions on Control Systems Technology, vol. 21, no. 1, 2013.

[43] M. Bazrafshan and N. Gatsis, "Comprehensive Modeling of ThreePhase Distribution Systems via the Bus Admittance Matrix," IEEE Transactions on Power Systems, vol. 33, no. 2, pp. 2015-2029, 2018.

[44] S. Haben, G. Giasemidis, F. Ziel, and S. Arora, "Short Term Load Forecasting and the Effect of Temperature at the Low Voltage Level," International Journal of Forecasting, vol. 35, no. 4, pp. 1469-1484, 2018.

[45] S. Franceschini, C. Tsai, and M. Marani, "Point Estimate Methods Based on Taylor Series Expansion - The Perturbance Moments Method - A More Coherent Derivation of the Second Order Statistical Moment," Applied Mathematical Modelling, vol. 36, no. 11, pp. 5445-5454, 2012.

[46] L. Dong, W. Wang, and S. Lin, "Probabilistic Calculation of Locational Marginal Price Taking Account of Correlated Loads," in Asia-Pacific Power and Energy Engineering Conference, 2012, pp. 1-4.
[47] X. Zhang and S. Grijalva, "A Data-Driven Approach for Detection and Estimation of Residential PV Installations," IEEE Transactions on Smart Grid, vol. 7, no. 5, pp. 2477-2485, 2016.

[48] AEMO, "DER Register Information Guidelines," Tech. Rep., 2019. [Online]. Available: aemo.com.au/Electricity/National-ElectricityMarket-NEM/DER-program/DER-Register-Implementation

[49] Energy Systems Catapult, "Asset Registration Strategy," Tech. Rep., 2019. [Online]. Available: es.catapult.org.uk

[50] T. Fleiner, Z. Janko, A. Tamura, and A. Teytelboym, "Trading Networks with Bilateral Contracts," in The Third Conference on Auctions, Market Mechanisms and Their Applications, 2015, pp. 1-39.

[51] "European Low Voltage Test Feeder." [Online]. Available: http://sites.ieee.org/pes-testfeeders

[52] Customer-Led Network Revolution, "Enhanced Profiling of Domestic Customers With Solar Photovoltaics." [Online]. Available: networkrevolution.co.uk

[53] "Electric Power Systems and Equipment - Voltage Ratings $(60 \mathrm{~Hz})$ ANSI/NEMA C84.1," 2006. [Online]. Available: nema.org/Standards

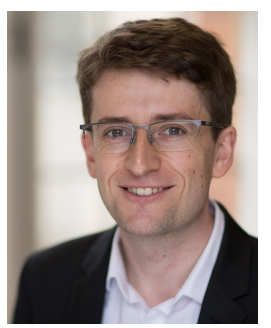

Thomas Morstyn (S'14-M'16) received the BEng (Hon.) degree from the University of Melbourne in 2011, and the $\mathrm{PhD}$ degree from the University of New South Wales in 2016, both in electrical engineering.

$\mathrm{He}$ is an EPSRC Research Fellow with the Department of Engineering Science at the University of Oxford, and he is a fellow with the Oxford Martin Programme on Integrating Renewable Energy. His research interests include multi-agent control and market design for integrating distributed energy resources into power system operations.

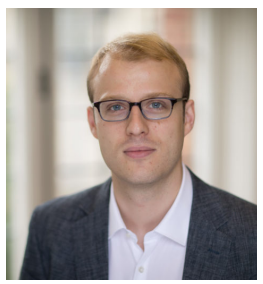

Alexander Teytelboym received a B.Sc. in Economics (2008) from the L.S.E. and M.Phil. (2010) and D.Phil. in Economics (2013) from the University of Oxford.

$\mathrm{He}$ is an economist interested in market design as well as social and economic networks. Before joining the Department of Economics, he was the Otto Poon Research Fellow at the Institute for New Economic Thinking at the Oxford Martin School and prior to that a Postdoctoral Fellow at the Laboratory for Information and Decision Systems at M.I.T.

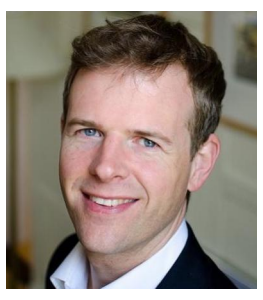

Cameron Hepburn received the BEng (Chem.) and LLB (Hon.) degrees from the University of Melbourne in 1999, and M.Phil. and D.Phil. in Economics from the University of Oxford in 2005.

$\mathrm{He}$ is Professor of Environmental Economics at the University of Oxford, and Director of the Smith School of Enterprise and the Environment. He also serves as the Director of the Economics of Sustainability Programme, based at the Institute for New Economic Thinking at the Oxford Martin School. He has published widely on energy, resources and environmental challenges across disciplines including engineering, biology, philosophy, economics, public policy and law.

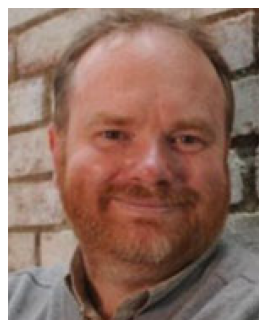

Malcolm D. McCulloch (SM'89) received the B.Sc. (Eng.) and Ph.D. degrees in electrical engineering from the University of the Witwatersrand, Johannesburg, South Africa, in 1986 and 1990, respectively.

In 1993, he joined the University of Oxford, Oxford, U.K., to head up the Energy and Power Group, where he is currently an Associate Professor in the Department of Engineering Science. He is active in the areas of electrical machines, transport, and smart grids. His work addresses transforming existing power networks, designing new power networks for the developing world, developing new technology for electric vehicles, and developing approaches to integrated mobility. 